\title{
Efficient and robust numerical modeling of variably saturated flow in layered porous media
}

\author{
C. T. Miller \& G. A. Williams \\ Department of Environmental Sciences and Engineering, \\ University of North Carolina, Chapel Hill, NC \\ EMail: casey_miller@unc.edu,glenn_williams@unc.edu
}

\section{Abstract}

We extend our recent work on higher-order time integration of Richards' equation to layered heterogeneous porous media, using a differential-algebraicequation-based method of lines (DAE/MOL) approach. We show that the DAE/MOL approach is robust and efficient compared to standard low-order time integration methods for heterogeneous media. We also show the advantage of using an integral representation of permeability compared to a standard arithmetic mean for the test problems considered herein.

\section{Introduction}

Richards' equation (RE) is commonly used to describe flow in partially saturated porous media [], although questions about the validity of this approach remain []. RE is commonly solved using low-order spatial approximations [] and low-order temporal-integration methods []; several codes are available that implement these methods [].

In our recent work, we have shown: (1) DAE/MOL approaches for solving $\mathrm{RE}$ are robust and more efficient than traditional loworder approaches [?]; (2) modification to a standard DAE integrator can further improve efficiency [?]; (4) use of vectorizable interpolation methods for evaluating constitutive relations can drammatically affect simulator performance [?]; (3) monitoring of the condition number of the Jacobian is an effective strategy to aid in the selection 
of nonlinear termination criteria [?]; (5) lack of smoothness in van Genuchten-Mualem relations for common media conditions can lead to problems with convergence of nonlinear solvers - a situation that can be remedied using an integral conductivity representation and cubic spline interpolation []; (6) DAE/MOL approaches generalize to two dimension, as does condition-number-based termination of the nonlinear solver []; and (7) transformation approaches can significantly improve the efficiency of RE solution methods for both standard low-order methods and DAE/MOL approaches [].

While these advances have contributed to the robustness and efficiency of solutions for RE, the focus of this work has been on homogeneous media, except our recent investigation of a slightly heterogeneous two-dimensional system in which the focus was on linear and nonlinear solver issues []. Neither our previous work, nor the work of others that we are aware of have investigated the efficiency and robustness of the DAE/MOL approach for solving RE for heterogeneous media, although such conditions are typical of natural systems. This seems especially important to consider for media that lacks smoothness in certain situations.

The objective of this work is to compare standard, low-order methods for solving RE to a DAE/MOL approach for heterogeneous media conditions. This comparison has two important components: robustness, or the reliability of the solution method; and efficiency, the error in the solution achieved for a given investment in computational resources.

\section{Methods}

\subsection{Formulation}

We consider two common forms of RE in one spatial dimension, the pressure-head-based form

$$
\left[c(\psi)+S_{s} S_{a}(\psi)\right] \frac{\partial \psi}{\partial t}=\frac{\partial}{\partial z}\left[K(\psi)\left(\frac{\partial \psi}{\partial z}+1\right)\right]
$$

and the mixed form

$$
\frac{\partial \theta_{a}}{\partial t}+S_{s} S_{a}(\psi) \frac{\partial \psi}{\partial t}=\frac{\partial}{\partial z}\left[K(\psi)\left(\frac{\partial \psi}{\partial z}+1\right)\right]
$$


where $c=d \theta_{a} / d \psi$ is the specific moisture capacity, $S_{s}$ is the specific storage coefficient, which accounts for fluid compressibility; $S_{a}$ is saturation of the aqueous phase; $\psi$ is the pressure head; $t$ is time; $\theta_{a}$ is the volumetric fraction of the aqueous phase; $z$ is the vertical spatial dimension; and $K$ is the hydraulic conductivity.

We consider problems with auxiliary conditions of the form

$$
\begin{aligned}
\psi(z, t=0) & =\psi_{0}(z) \\
\psi(z=0, t>0) & =\psi_{1} \\
\psi(z=Z, t>0) & =\psi_{2}
\end{aligned}
$$

where $Z$ is the length of the domain, $\psi_{0}$ may be a function of space, and $\psi_{1}$ and $\psi_{2}$ are constants. These conditions lead to the development of a sharp infiltration front and saturated conditions over a portion of the domain, which is a difficult class of test problem.

For closure, we use the standard van Genuchten (VG) pressuresaturation relationship [van Genuchten(1980), ], which is given by

$$
S_{e}(\psi)=\frac{\theta_{a}(\psi)-\theta_{r}}{\theta_{s}-\theta_{r}}= \begin{cases}\left(1+\left|\alpha_{v} \psi\right|^{n_{v}}\right)^{-m_{v}}, & \psi<0 \\ 1, & \psi \geq 0\end{cases}
$$

and the Mualem saturation-conductivity relation $[\operatorname{Mualem}(1976)$,

$$
K\left(S_{e}\right)=K_{s} S_{e}^{1 / 2}\left[1-\left(1-S_{e}^{1 / m_{v}}\right)^{m_{v}}\right]^{2}
$$

where $m_{v}=1-1 / n_{v}, S_{e}$ is the effective saturation, $\theta_{r}$ is the residual volumetric water content, $\theta_{s}$ is the saturated volumetric water content, $\alpha_{v}$ is a parameter related to the mean pore size, $n_{v}$ is a parameter related to the uniformity of the pore-size distribution, and $K_{s}$ is the water-saturated hydraulic conductivity.

\subsection{Spatial Discretization}

We use a standard finite-difference approximation to discretize RE with respect to the spatial dimension [Celia et al.(1990)Celia, Bouloutas, and Zarba, ], $z$, where $z \in[0, Z]$. We consider a uniform spatial discretization comprised of $n_{n}-1$ intervals $\left\{\left[z_{i}, z_{i+1}\right]\right\}_{i=1}^{n_{n}-1}$, of length $\Delta z$, with $\Delta z=Z /\left(n_{n}-1\right)$, and $z_{i}=(i-1) \Delta z$ for $1 \leq i \leq n_{n}$. The spatial operator

$$
O_{s d}(\psi)=\frac{\partial}{\partial z}\left[K(\psi)\left(\frac{\partial \psi}{\partial z}+1\right)\right]
$$


is approximated at $z=z_{i}$ for $1<i<n_{n}$ by

$$
\begin{aligned}
O_{s d_{i}}(\psi)= & \Delta z^{-2}\left[K_{i+1 / 2}\left(\psi_{i+1}-\psi_{i}\right)-K_{i-1 / 2}\left(\psi_{i}-\psi_{i-1}\right)\right] \\
& +\Delta z^{-1}\left(K_{i+1 / 2}-K_{i-1 / 2}\right)
\end{aligned}
$$

where $n_{n}$ is the number of spatial nodes in the solution, and $\psi_{i}$ is the approximation to $\psi\left(z_{i}\right)$.

Interblock conductivities, $K_{1 \pm 1 / 2}$ are evaluated using an arithmetic mean (KAM)

$$
K_{i \pm 1 / 2}=\left(K_{i}+K_{i \pm 1}\right) / 2
$$

and an integral (KINT) approximation

$$
K_{i \pm 1 / 2}= \begin{cases}\frac{1}{\left|\psi_{i}-\psi_{i \pm 1}\right|} \int_{\min \left\{\psi_{i}, \psi_{i \pm 1}\right\}}^{\max \left\{\psi_{i}, \psi_{i \pm}\right\}} K d, & \text { if } \psi_{i} \neq \psi_{i \pm 1} \\ K\left(\psi_{i}\right), & \text { if } \psi_{i}=\psi_{i \pm 1}\end{cases}
$$

\subsection{Temporal Integration}

We considered two temporal integration methods, the first method is a standard implicit finite difference approximation applied to the mixed-form equation and solved using modified Picard iteration (MPI), which is considered the standard approach []. Time step size was selected using a common empirical adaptive time-step control algorithm [Rathfelder and Abriola(1994), ]

- if $m<m_{l}$ then $\Delta t_{n+1}=\min \left(f_{t} \Delta t_{n}, \Delta t_{\text {max }}\right)$

- else if $m>m_{u}$ then $\Delta t_{n+1}=\max \left(\Delta t_{n} / f_{t}, \Delta t_{\text {min }}\right)$

where $m$ is the number of iterations required by the nonlinear solver to converge for time step $n, m_{l}$ is a lower iteration limit, $m_{u}$ is an upper iteration limit, $f_{t}$ is a time-step acceleration factor, $\Delta t_{\max }$ is the maximum allowable time-step size, and $\Delta t_{\min }$ is the minimum allowable time-step size.

The second time integration method investigated was a DAE/ MOL approach for the pressure-head form of RE (1), which was applied to the semi-discrete form of $\mathrm{RE}$ given by

$$
A(\psi)_{i} \frac{d \psi_{i}}{d t}=O_{s d i}(\psi)
$$


where $A$ includes accumulation and compressibility terms. A modified version of DASPK [Brown et al.(1994)Brown, Hindmarsh, and Petzold, Tocci et al.(1997)Tocci, Kelley, and Miller, ] was used as our DAE solver. DASPK is a DAE solver based upon the fixed leading coefficient, first- through fifth-order backward difference formulas and containing error estimation and control through adjustment of method order and step size [Brenan et al.(1996)Brenan, Campbell, and Petzold, ].

\subsection{Efficiency}

We define efficiency as the computational effort required to achieve a specified accuracy, which requires evaluation of both work and errorthe former being challenging and the latter trivial. We evaluate work for the MPI method by

$$
W_{p}=w_{c} n_{c}+w_{l} n_{l}
$$

and for the DAE/MOL method by

$$
W_{n}=w_{j} n_{j}+w_{f} n_{f}+w_{l} n_{l}
$$

where $W_{p}$ is a work measure for MPI methods, $w_{c}$ is a weighting factor for formation of the coefficient matrix and right hand side vector, which are typically done at the same time, $w_{l}$ is a weighting factor for solution of the linear system of equations, $n_{c}$ is the number of coefficient matrix formation calls, $n_{l}$ is the number of linear solutions performed, $W_{n}$ is a work measure for Newton iteration DAE methods, $w_{j}$ is a weighting factor for formation of the Jacobian matrix, $w_{f}$ is a weighting factor for evaluation of the function, $n_{j}$ is the number of Jacobian evaluations, and $n_{f}$ is the number of function evaluations. We estimated these weights based upon CPU time on a HewlettPackard ... running version ... of ... operating system, and using version - of $\ldots$ complier as $w_{c}=0.484, w_{j}=0.552, w_{f}=0.271$, and $w_{l}=0.181$. Glenn these need to be updated.

Error was evaluated by comparison to dense-grid solutions using

$$
\left\|\epsilon_{D}\right\|_{k}=\left[\frac{1}{n_{n}} \sum_{i=1}^{n_{n}}\left(\left|\hat{\psi}_{i}-\psi_{i}\right|\right)^{k}\right]^{1 / k}
$$

where $k$ is the norm measure, with $k=1$ results are reported in this work; and $\hat{\psi}_{i}$ is an approximation of the true solution based on a 
Table 1: Media Properties

\begin{tabular}{lccc}
\hline Variable & Sand & Loam & Clay \\
\hline$\theta_{r}(-)$ & 0.093 & 0.078 & 0.102 \\
$\theta_{s}(-)$ & 0.301 & 0.430 & 0.368 \\
$\alpha_{v}\left(\mathrm{~m}^{-1}\right)$ & 5.470 & 3.600 & 3.350 \\
$n_{v}(-)$ & 4.264 & 1.560 & 2.000 \\
$K_{s}(\mathrm{~m} /$ day $)$ & 5.040 & 0.250 & 7.970 \\
$S_{s}\left(\mathrm{~m}^{-1}\right)$ & $1.0 \times 10^{-6}$ & $1.0 \times 10^{-6}$ & 0.000
\end{tabular}

dense spatial grid. The dense-grid solutions were generated using the DAE/MOL approach with a spatial grid size equal to $1 / 32$ of the size used in the test simulations. Glenn check this too.

\section{Results}

Two layered, heterogeneous test problems were investigated using the MPI and DAE/MOL approach described above. For both test problems, the spatial domain was $z \in[0,4] \mathrm{m}$. The temporal domains were $t \in[0,0.25]$ days for Problem 1 and $t \in[0,0.08]$ days for Problem 2. The media for Problem 1 consisted of four, 1-m thick alternating layers of loam and sand. The media for Problem 2 consisted of four 1-m thick alternating layers of clay and sand. The properties of these materials are listed in Table 1, which were taken from the literature []. Problem 1 exhibits a larger degree of heterogeneity than Problem 2 due to the wider difference in $K_{s}$ and $n_{v}$ between adjoining layers, making Problem 1 a more difficult test problem. The dense grid solution profile for both test problems is shown on Figure 1, which illustrates the effects of media heterogeneity and the sharp-front nature of the solution profiles.

Quantitative results in the form of work and error measures for each of the test problems are shown in Figures 2 and 3. The comparisons of the MPI and DAE/MOL approaches showed:

1. the DAE/MOL approach was more robust than the MPI approach, the MPI method failing to produce convergent solutions for Problem 1; 


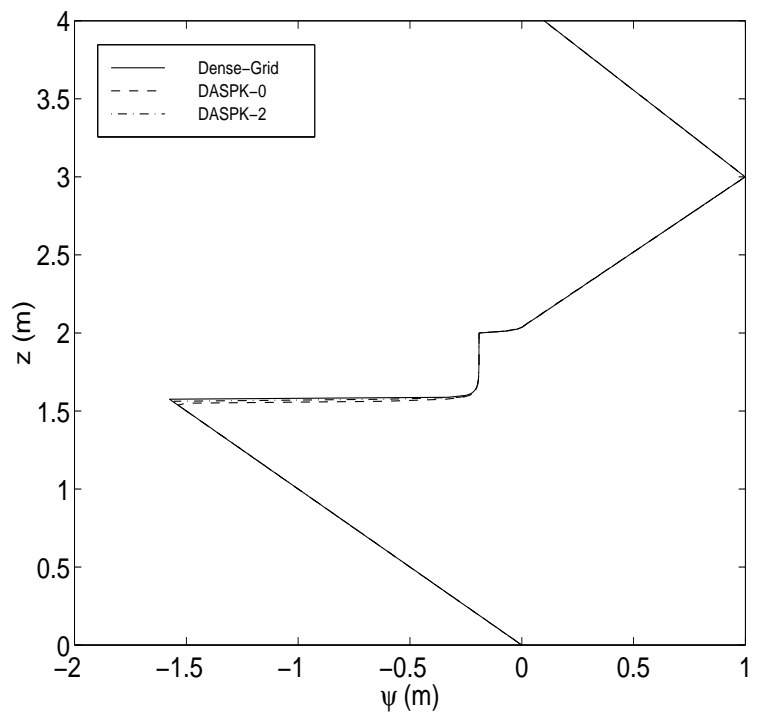

Figure 1: Solution Profiles for Test Problems 1 and 2.

2. the DAE/MOL approach was more efficient than the MPI approach when both methods converged, producing equivalent accuracy results with as little as $30 \%$ of the computational effort in one case; and Glenn check this.

3. the KINT produced more accurate and more efficient results than the KAM approach. Glenn again check this.

\section{Acknowledgments}

This work was supported in part by U.S. Army Waterways Experiment Station Contract DACA39-95-K-0098, Army Research Office Grant DAAL03-92-G-0111, National Institute of Environmental Health Sciences Grant 5 P42 ES05948. Computing activity was partially supported by allocations from the North Carolina Supercomputing Center. 


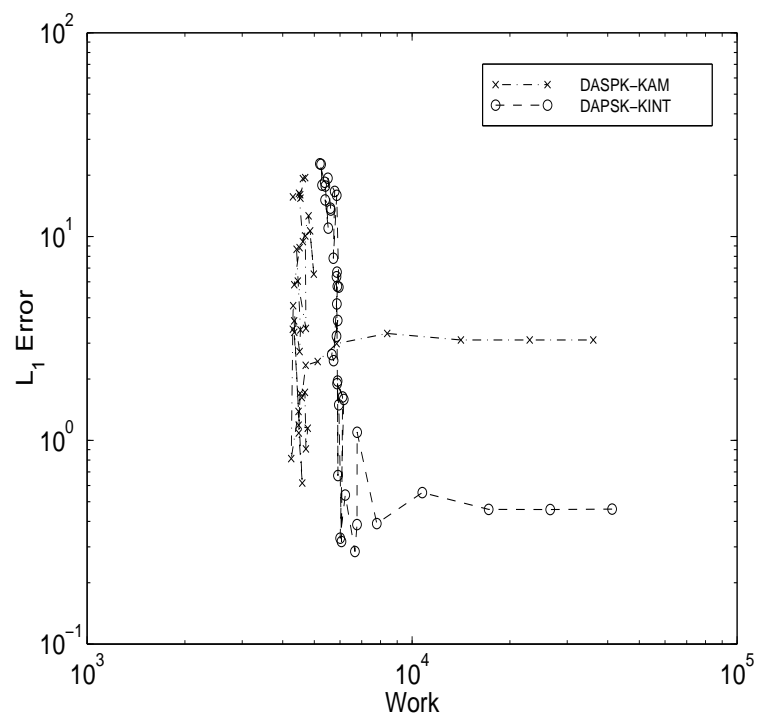

Figure 2: Solution Profile for Test Problem 1.

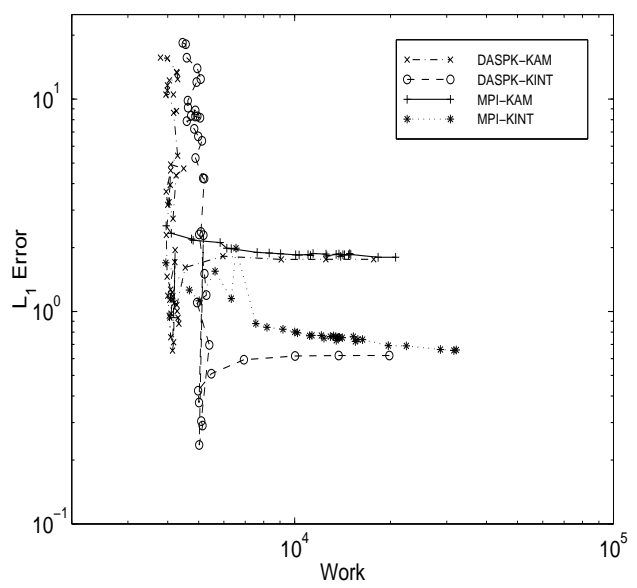

Figure 3: Solution Profile for Test Problem 2. 


\section{References}

[Brenan et al.(1996)Brenan, Campbell, and Petzold] Brenan, K. E., S. L. Campbell, and L. R. Petzold, The Numerical Solution of Initial Value Problems in Differential-Algebraic Equations, vol. 16 of SIAM Frontiers in Applied Mathematics, SIAM, Philadelphia, PA, 1996.

[Brown et al.(1994)Brown, Hindmarsh, and Petzold] Brown, P. N., A. C. Hindmarsh, and L. R. Petzold, Using Krylov methods in the solution of large-scale differential-algebraic systems, SIAM J. Stat. Comp, 15, 1467-1488, 1994.

[Celia et al.(1990)Celia, Bouloutas, and Zarba] Celia, M. A., E. T. Bouloutas, and R. L. Zarba, A general mass-conservative numerical solution for the unsaturated flow equation, Wat. Resour. Res., 26(7), 1483-1496, 1990.

[Mualem(1976)] Mualem, Y., A new model for predicting the hydraulic conductivity of unsaturated porous media, Wat. Resour. Res., 12, 513-522, 1976.

[Rathfelder and Abriola(1994)] Rathfelder, K. and L. M. Abriola, Mass conservative numerical solutions of the head-based Richards' equation, Wat. Resour. Res., 30(9), 2579-2586, 1994.

[Tocci et al.(1997)Tocci, Kelley, and Miller] Tocci, M. D., C. T. Kelley, and C. T. Miller, Accurate and economical solution of the pressure-head form of richards' equation by the method of lines, Adv. in Wat. Resour., 20(1), 1-14, 1997.

[van Genuchten(1980)] van Genuchten, M. T., A closed-form equation for predicting the hydraulic conductivity of unsaturated soils, Soil Sci. Am. J., 44, 892-898, 1980. 